\title{
Feshbach Resonance and Growth of a Bose-Einstein Condensate
}

\author{
C. Yuct ${ }^{*}$ and A. Kilic \\ Department of Physics, Anadolu University, Eskisehir, Turkey
}

(Dated: April 9, 2018)

\begin{abstract}
Gross-Pitaevskii equation with gain is used to model Bose Einstein condensation (BEC) fed by the surrounding thermal cloud. It is shown that the number of atoms continuously injected into BEC from the reservoir can be controlled by applying the external magnetic field via Feshbach resonance.

PACS numbers: 03.75.-b, 03.75.Kk, 05.45.Yv
\end{abstract}

\section{INTRODUCTION}

Gross-Pitaevskii (GP) equation is one of the most important nonlinear equations used in physics. It appears in many branches of physics such as nonlinear optics, fluid mechanics, condensed matter and theory of superfluidity. For example, GP equation is used to explore the macroscopic behavior of the Bose-Einstein condensation (BEC) at low temperature. Its validity in the BEC is based on the condition that the s-wave scattering length should be much smaller than the average distance between the atoms and that the number of atoms should be much larger than one. Experimental achievements and theoretical studies of Bose-Einstein condensates (BEC) of weakly interacting atoms have stimulated intensive interest in the field of atomic matter waves and nonlinear excitations such as dark [1, 2, 3] and bright solitons [4, 5, 6, 7]. The dynamics of BEC crucially depends on the sign of interatomic interaction. Dark (bright) solitons that are solutions to the GP equation can be generated with repulsive (attractive) interactions, resulting from the positive (negative) s-wave scattering length. In the present study, we start by considering the Gross-Pitaevskii (GP) equation modified by a linear term. The Gross-Pitaevskii gain equation reads;

$$
i \hbar \frac{\partial \Psi}{\partial t}=-\frac{\hbar^{2}}{2 m} \nabla^{2} \Psi+V_{t r a p} \Psi+\frac{4 \pi \hbar^{2}}{m} a_{s}|\Psi|^{2} \Psi+i \hbar \frac{\Gamma}{2} \Psi
$$

where $V_{\text {trap }}=\frac{m}{2} \omega_{\perp}^{2}\left(x^{2}+y^{2}\right)+\frac{m}{2} \omega_{z}^{2} z^{2}, a_{s}(t)$ is the time-dependent scattering length and the constant $\Gamma$ is the gain (loss) term for $\Gamma>0(\Gamma<0)$. The complex linear term with positive $\Gamma$ accounts for the mechanism of loading the thermal cloud into the BEC by optically pumping atoms from the external cold atomic-beam source. From the physical point of view, replenishing is required in steady state to compensate for various intrinsic loss mechanisms, such as collisions with hot atoms from the background vapor and inelastic two and three-body collisions between the trapped atoms. However, for the negative values of $\Gamma$, the above equation describes a BEC that is continuously depleted by loss.

For attractive interaction, the number of atoms in the condensate grows until the total number of atoms in the condensate exceeds a critical value with which the BEC undergoes collapse. During the collapse, the number of atoms is decreased. If the condensate is fed by a surrounding thermal cloud, then the condensate undergoes cycles of growth and collapses. Hulet and his team at Rice university observed the growth of a condensate of trapped ${ }^{7} \mathrm{Li}$ atoms with attractive interaction and its subsequent collapse [8]. Ketterle with his team measured the rate of growth of a ${ }^{23} \mathrm{Na}$ condensate fed by a thermal cloud 9]. Growth of a Bose-Einstein condensate from thermal vapor was also experimentally realized for atoms of ${ }^{87} \mathrm{Rb}[10]$. Some methods have been introduced theoretically to account for the growth of the BEC. GP gain equation was used to model the growth of a BEC by Drummond and Kheruntsyan [1]. They determined that, as the condensate grows, the center of mass oscillates in the trap.

Furthermore, GP gain equation is of special importance in the field of an atom laser, that is a device which produces an intense coherent beam of atoms by a stimulated process $[12,13$.

In recent years, GP gain equation (11) has also been studied mathematically by some authors 14, 15, 16]. GP gain equation can also be used in the theory of optical fibers if the harmonic trap potential is assumed to be zero [15, 16]. The interaction effect in the system (11) is determined by the s-wave scattering length. In ref. 17], it has been shown that controlling the generation of bright and dark soliton trains from periodic waves can be achieved by the variation of the scattering length. Recent experiments have demonstrated that tuning of the s-wave scattering length can be achieved due to the Feshbach resonance 18, 19, 20, 21, 22]. It offers a possibility to vary the interaction strength in ultracold atomic gases simply by applying an external magnetic field.

$$
a_{s}(t)=a\left\{1+\Delta /\left[B_{0}-B(t)\right]\right\},
$$

where $a$ is the asymptotic value of the scattering length far from resonance, $B(t)$ is the time-dependent externally applied magnetic field, $\Delta$ is the width of resonance and $B_{0}$ is the resonant value of the magnetic field. The strength 
of the interaction can be adjusted experimentally from large negative values to large positive values. As a result, the experiments with magnetically trapped ultra cold atomic gases, where the s-wave scattering length fully determines the interaction effects, have an unprecedented high level of control over the interatomic interactions. With this experimental degree of freedom, it is possible to intensively study the dynamics of BEC.

Here, we will study the changes in the number of atoms supplied by a surrounding thermal cloud due to the variations of the scattering length by the external magnetic field. We will assume that $\Gamma$ does not change but the s-wave scattering length changes via Feshbach resonance.

By supposing the number of particles is not very large for the cigar-shaped traps, it is legitimate to use the reduced 1D GP equation

$$
i \frac{\partial \Psi}{\partial t}=-\frac{1}{2} \frac{\partial^{2} \Psi}{\partial z^{2}}+\frac{\omega^{2}}{2} z^{2} \Psi+\frac{2 a_{s}}{a_{B}}|\Psi|^{2} \Psi+i \frac{\gamma}{2} \Psi
$$

where $\omega^{2}=\omega_{z}^{2} / \omega_{\perp}^{2}, \Gamma=\gamma / \omega_{\perp}$ and $a_{B}$ is the Bohr radius. Here, the coordinate $z$ and the time $t$ are measured in units of $a_{\perp}$ and $1 / \omega_{\perp}$, respectively. The relationship between the macroscopic wave functions $\Psi(\vec{r}, t)$ and $\Psi(z, t)$ is given by

$$
\Psi(\vec{r}, t)=\frac{1}{\sqrt{2 \pi a_{B}} a_{\perp}} \exp \left(-i \omega_{\perp} t-\frac{x^{2}+y^{2}}{2 a_{\perp}^{2}}\right) \times \Psi\left(\frac{z}{a_{\perp}}, \omega_{\perp} t\right)
$$

where $a_{\perp}=\left(\hbar / m \omega_{\perp}\right)^{1 / 2}$. To extract qualitative information and to improve the understanding of the underlying physics, we will transform the GP gain equation (3) analytically to the one with the constant scattering length.

To get rid of the time-dependent scattering length from the equation (3), the two transformations are introduced as follows;

$$
\Psi(z, t) \rightarrow f(t)^{-1 / 2} \exp \left(\int \frac{\gamma\left(f^{2}-1\right)}{f^{2}} d t^{\prime}+\frac{i \dot{f}}{2 f} z^{2}\right) \Phi(z, t) ; \quad z \rightarrow f(t) z,
$$

where dot denotes time derivation and the time-dependent function $f(t)$ is given by

$$
f(t)=\sqrt{\frac{\sin 2 \omega t+\sqrt{1+\Omega^{2}}}{\omega}}
$$

where $\Omega$ is a constant. Note that the time-dependent function $f(t)$ is chosen in such a way that it satisfies $f^{3} \ddot{f}+\omega^{2} f^{4}=\Omega^{2}$. The scale transformation on the coordinate accounts for the contraction or the expansion of the condensate depending on the behavior of $f(t)$. The time-dependent scaling of the coordinate and re-scaling of the wave function have been previously applied to harmonic oscillator potential with the time-dependent angular frequency $\omega^{2}(t)[23,24,25]$. These scale transformations are used here to explore the effect of Feshbach resonance on the number of atoms injected into BEC.

Now, let us first substitute the transformation for $\Psi$ and then for the coordinate into the equation (3). Note that under the coordinate scaling, the time derivative operator transforms as $\partial / \partial t \rightarrow \partial / \partial t-(\dot{f} / f) z \partial / \partial z$. We observe that the equation (31) can be reduced to the another GP gain equation with the constant scattering length

$$
i \frac{\partial \Phi}{\partial \tau}=-\frac{1}{2} \frac{\partial^{2} \Phi}{\partial z^{2}}+\frac{\Omega^{2}}{2} z^{2} \Phi+\frac{2 a}{a_{B}}|\Phi|^{2} \Phi+i \frac{\gamma}{2} \Phi
$$

where $a$ is a constant scattering length. We made another transformation on time $\tau=\int_{0}^{t} f^{-2} d t^{\prime}$ in the last step. The necessary and sufficient condition for the existence of such a reduction is given by $a_{s}(t)=a f^{-1} \exp \left(-2 \int \frac{\gamma\left(f^{2}-1\right)}{f^{2}} d t^{\prime}\right)$. As a special case, if $f^{2}>>1$ for some special values of $\omega^{2}$ and $\Omega^{2}$, then $a_{s}(t)$ can be approximated as $a_{s}(t) \approx a f^{-1} e^{-2 \gamma t}$.

The magnitudes of trapping frequencies $(\Omega, \omega)$ are scaled. We have freedom to make them equal by choosing $\Omega=\omega$ or to turn-off the trap by choosing $\Omega=0$.

It is interesting to observe that the equation (3) can be transformed into the another GP gain equation with a different time parameter (7). The two equations (317) describe the two different condensates continuously replenished by pumping from the reservoir. The only physical difference between the two condensates is that the latter one is with the constant scattering length while the former one is with Feshbach resonance. The transition from one to the other 
is given by the transformations (5).

This reduction is of great importance in the sense that although the corresponding exact solutions are not known, it allows us to understand the effect of Feshbach resonance on the dynamics of the condensates.

Let us find how the Feshbach resonance changes the number of atoms supplied to the system from the reservoir by using (5). Note that the gain coefficient $\gamma$ doesn't change for the two condensates.

$$
\int|\Psi|^{2} d^{3} r=\exp \left(\int \frac{\gamma\left(f^{2}-1\right)}{f^{2}} d t^{\prime}\right) \int|\Phi|^{2} d^{3} r .
$$

This formula specifically shows the effect of the time-dependent nature of the scattering length on the number of atoms in the condensates. The relation (8) has been derived without obtaining the exact solution to the GP gain equation. It is concluded that simply by applying the external magnetic field, one can control the number of atoms transferred from the surrounding cloud. In an experiment for the negative sign of $\gamma$, one can also take advantage of Feshbach resonance to control the number of atoms left in the BEC.

The method applied here is analytical and only works for a special $a_{s}(t)$. Numerical calculations can be performed for the most general $a_{s}(t)$ on the basis of the fact that Feshbach resonance can be used to control the number of atoms supplied to the system.

As a special case, if we set $\gamma=0$, then the number of atoms doesn't change for the two BECs (317) as expected. In other words, Feshbach resonance does not change the number of atoms if there is no reservoir. The transformations (5) conserve the total number of atoms. However, the presence of $f(t)$ in the transformations (5D) has the effect of increasing the central peak of the condensate with a consequent contraction of the atomic cloud.

If $\gamma \neq 0$, the number of particles as well as the condensate size is changed by Feshbach resonance as can be seen from the equation (8). The transformations (5) allow us to understand the underlying physical effects of time-dependent nature of the scattering length on GP gain equation, which is used to model the condensate growth and atom laser. Let us study the evolution of the system if we choose $\Omega=0$. In this case, the equation (7) describes a BEC with the trap potential turned-off and continuously replenished by pumping from the reservoir. However, the equation (3) describes another BEC whose scattering length changes with time through the application of the external magnetic field. Atoms are injected into both condensates by the same gain coefficient $\gamma$. Since the harmonic potential does not exist and the scattering length is constant in (7), its solution can be constructed in terms of the well-known bright and dark solitons depending on the sign of the scattering length. Then, the exact solution for (3) can also be constructed by using the analytical transformations given in (5).

The time parameter transforms as $\tau=\frac{\sin \omega t}{\sin \omega t+\cos \omega t}$ when $\Omega=0$. The transformations (5) works in the time interval $0 \leq \omega t<3 \pi / 4$. In this interval, $f(t)=\sqrt{(1+\sin 2 \omega t) / \omega}$ varies from $\sqrt{1 / \omega}$ to zero. Switching-off the trap potential has dramatic effects on the dynamics of the system. The longitudinal width of the two condensates can be compared by making use of the coordinate transformation $(z \rightarrow z \sqrt{(1+\sin 2 \omega t) / \omega})$. If it is equal to $L$ for the BEC described by (7), then it is equal to $L \sqrt{(1+\sin 2 \omega t) / \omega}$ for the other BEC described by (3). The time $\omega t=\pi / 4$ is a turning point in the sense that the longitudinal width of the BEC starts to decrease from this time while it is increasing in the interval $0 \leq \omega t \leq \pi / 4$.

The condensate collapses into itself at time $\omega t=3 \pi / 4$ since the longitudinal width of the BEC (3) goes to zero. Furthermore, the density of the BEC shows asymptotic behavior depending on the sign of $\gamma$. At time $\omega t=3 \pi / 4$, $|\Psi|^{2} \rightarrow \infty|\Phi|^{2}$ for $\gamma>0$ while the longitudinal width of the BEC (3i) approaches zero. The matter wave function becomes somewhat like a Dirac delta function. This is reasonable, since atoms are constantly injected into the condensate. However, this is not physical, because the BEC becomes a singular point. From the physical point of view, GP gain equation is not valid for the description of the BEC if the density of the BEC is big enough. To prevent the formation from being a true singularity, the three-body recombination losses are expected to cause rapid depletion of the BEC. In order to model atom loss due to 3-body recombination, a phenomenological term proportional to $\Psi^{4}$ should be added to the GP gain equation (1) for a good physical description of the BEC. Note also that, the scattering length $a_{s}(t)$ goes to zero if $\gamma>0$ at this time. Physically, the scattering length can be made zero by tuning the external magnetic field. For example, at a magnetic field of $B \approx 166.5 \mathrm{G}$, the scattering length vanishes for ${ }^{85} \mathrm{Rb}$ and the gas behaves effectively as an ideal Bose gas. However, for the negative values $(\gamma<0)$, $|\Psi|^{2} \rightarrow 0|\Phi|^{2}$ at time $\omega t=3 \pi / 4$. Since the atoms are lost for the negative values of $\gamma$, both the longitudinal width and the number of atoms get smaller in time. The scattering length $a_{s}(t)$ goes to infinity if $\gamma<0$ at this time.

Let us now study the case if $\Omega^{2}>0$. The equations (317) describe two condensates in the confining harmonic traps with two different trap frequencies $\omega^{2}, \Omega^{2}$. The scattering length is time-dependent for the former, while it is constant for the latter. Both of them are continuously replenished by pumping from the reservoir with the same gain coefficient.

The relation between the time parameters is given by $\Omega \tan \Omega \tau=1+\sqrt{1+\Omega^{2}} \tan \omega t$, where $0 \leq \omega t<\pi / 2 . f(t)$ is increasing in the interval $0 \leq t \leq \pi / 2$ and decreasing in the interval $\pi / 2<t<\pi / 4$. In this case, $f(t)$ does not 
become zero. Then, the singularity in the BEC doesn't occurs in this case. This is because of the existence of the harmonic trapping potential. The harmonic potential which has zero point energy in the ground state plays a central role in preventing the condensate from being singularity.

In conclusion, although the atoms are loaded with the same gain coefficients, the time dependent nature of the scattering length changes the number of atoms transferred to the BEC from the reservoir as can be seen from (8). The transformations introduced here provides for an analytic way to investigate the effect of Feshbach resonance on the number of atoms which are continuously loaded or depleted by loss.

* Electronic address: cyuce@anadolu.edu.tr

[1] S. Burger et al., Phys. Rev. Lett. 83, 5198 (1999).

[2] J. Denschlag et al., Science 287, 97 (2000).

[3] B. P. Anderson et al., Phys. Rev. Lett. 86, 2926 (2001).

[4] K. E. Strecker et al., Nature (London) 417, 150 (2002).

[5] L. Khaykovich et al., Science 296, 1290 (2002).

[6] K. D. Moll, A. L. Gaeta, G. Fibich, Phys. Rev. Lett. 90, 203902 (2003).

[7] S. J. Wang, C. L. Jia, D. Zhao, H. G. Luo, J.H. An, Phys. Rev. A 68, 015601 (2003).

[8] J. M. Gerton et al., Nature 408, 692 (2000).

[9] H. J. Miesner et al.,Science 279, 1005 (1998).

[10] M. Kohl, M. J. Davis, C. W. Gardiner, T. W. Hansch, T. Esslinger, Phys. Rev. Lett. 88, 080402 (2002).

[11] P. D. Drummond, K. V. Kheruntsyan, Phys. Rev. A. 63, 013605 (2001).

[12] B. Kneer, T. Wong, K. Vogel, W. P. Schleich, D. F. Walls, Phys. Rev. A. 58, 4841 (1998).

[13] A. M. Guzman, M. Moore, and P. Meystre, Phys. Rev. A 53, 977 (1996).

[14] R. Atre, P.K. Panigrahi, G. S. Agarwal, Phys. Rev. E 73056611 (2006).

[15] V. N. Serkin and A. Hasegawa, Phys. Rev. Lett. 85, 4502 (2002).

[16] V. I. Kruglov, A. C. Peacock, J. D. Harvey, Phys. Rev. Lett. 90, 113902 (2003).

[17] F. K. Abdullaev, A. M. Kamchatnov, V. V. Konotop, V. A. Brazhnyi, Phys. Rev. Lett. 90, 230402 (2003).

[18] A. J. Moerdijk, B. J. Verhaar, A. Axelsson, Phys. Rev. A 51, 4852 (1995).

[19] J. L. Roberts et al., Phys. Rev. Lett. 81, 5109 (1998).

[20] J. Stenger et al., Phys. Rev. Lett. 82, 2422 (1999).

[21] S. L. Cornish, N. R. Claussen, J. L. Roberts, E. A. Cornell, C. E. Wieman, Phys. Rev. Lett. 85, 1795 (2000).

[22] P. G. Kevrekidis, G. Theocharis, D. J. Frantzeskakis, B. A. Malomed, Phys. Rev. Lett. 90, 230401 (2003).

[23] Y. Castin and R. Dum, Phys. Rev. Lett. 77, 5315 (1996).

[24] Y. Castin and R. Dum Phys. Rev. Lett. 79, 3553 (1997).

[25] Yu. Kagan, E. L. Surkov, and G. V. Shlyapnikov Phys. Rev. A 54, R1753 (1996). 